\title{
Unexpected challenges to preventing falls in older adults: a mixed methods study of an emergency department-based falls prevention referral pilot project
}

\author{
Suzanne FM van Wijck ${ }^{1,2}$, Caroline Rizzo ${ }^{3}$, Netsanet S Tsegai ${ }^{3}$, Toby Raybould ${ }^{1}$ and Shan W Liu ${ }^{3^{*}}$ \\ ${ }^{1}$ Division of Trauma, Emergency Surgery and Surgical Critical Care, Massachusetts General Hospital and Harvard Medical School, Boston, Massachusetts, USA \\ ${ }^{2}$ Department of Trauma Surgery, Leiden University Medical Center, Leiden, The Netherlands \\ ${ }^{3}$ Department of Emergency Medicine, Massachusetts General Hospital, Boston, MA, USA
}

\begin{abstract}
Objective: We sought to determine the rate and challenges to follow-up with a fall prevention program after an Emergency Department (ED) based referral.

Methods: We conducted a mixed-methods study of a pilot ED falls referral program. Older ( $\geq 65)$ patients who presented to the ED with fall-related injury were enrolled. We held semi-structured interviews in the ED and referred fall patients to an outpatient fall prevention program. We assessed quantitative outcomes, including 6-month mortality and recurrence of falls, and conducted follow-up phone interviews.

Results: One hundred geriatric patients patients were enrolled and 22 were referred. None (0\%) participated in the program. After 6 months, the mortality rate was $8.5 \%$ and $28 \%$ of the patients had recurrent falls. Qualitative data revealed that challenges to fall prevention were 1 ) practical considerations, 2 ) competing health issues, and 3) perceptions of fall risk.

Conclusion: None of our patients followed up in an outpatient falls program. Our patients had a high rate of recurrent falls. Follow-up interviews revealed that ED referral alone is not enough to overcome challenges to participation in a fall prevention clinic. Actively addressing barriers to fall clinic follow-up is necessary to improve outpatient fall clinic show rates.
\end{abstract}

\section{Introduction}

Falls are among the leading cause of injuries and deaths in older adults (aged 65 years and older) in the United States (USA). Each year more than 28,000 older adults die from falls and 2.8 million older adults visit the emergency department (ED) because of a fall [1]. Annual direct costs of non-fatal fall-related injuries in 2015 was estimated at $\$ 31$ billion and are expected to increase with an aging population $[1,2]$. Of all the older adults that visit the ED for fall related injuries, more than a third either returns to the ED or is deceased within a year [3].

Falls can have devastating and long-lasting consequences for older adults, decreasing overall functioning and lower quality of life. For community dwelling older adults, effective fall prevention strategies have the potential to significantly impact public health [4]. Because of the multifactorial etiology of falls, personalized fall prevention interventions that target multiple risk factors are most effective [5]. However, adherence to these prevention programs is consistently challenging [6]. Some of the known practical barriers include, but are not limited to, are costs, accessibility and time. Additionally, there are also psychological, social and cultural influences that result in different preferences for types of interventions. Being labelled as "at risk" for falling can lead to a fear of falling again and therefore lead to loss of independence and confidence. The effort to make sense of oneself in changed circumstances and the quality of the expertise of the healthcare provider about fall prevention and the patients' need and context, can influence the success of an intervention [7]. Another important part of a successful referral program is identifying the appropriate group of patients who would benefit $[8,9]$. Unfortunately, referral by primary care physicians often results in low adherence [8]. Also, referral of admitted patients, is hampered by many patients going to inpatient rehabilitation, making it impossible to attend an outpatient program upon hospital discharge.

However, there is a potential window of opportunity to identify the right group of patients in the ED for effective referral to fall prevention interventions $[10,11]$. Unfortunately, a previous study by Shankar, et al. [11], demonstrated that follow up in falls clinics was poor after an ED referral. However, little is known about why patients do not follow up after an ED referral. We sought to determine the rate of patient follow-up to a falls clinic after an ED fall clinic referral and what challenges existed to modifying fall risk in the following 6 months.

${ }^{\star}$ Correspondence to: Shan W Liu, Department of Emergency Medicine, Massachusetts General Hospital, MA, 55 Fruit Street, 5 Emerson, Room 119, Boston, MA 02114, USA, E-mail: sliu1@mgh.harvard.edu

Key words: falls, fall prevention, older adults, elderly patients, emergency department, follow-up, mixed methods

Received: June 28, 2019; Accepted: July 08, 2019; Published: July 11, 2019 


\section{Methods}

\section{Study design}

We conducted a mixed-methods pilot study in which we referred ED patients to a multidisciplinary outpatient fall prevention program. The program was a patient tailored, evidence-based prevention program centered on a rehabilitative model of care with a focus on enhancement of physical function, and was offered by a local rehabilitation center. Per the referral protocol, those who lived within a certain zip code close to the location of the program were referred. The other patients who lived outside these zip code areas served as a natural control group. As shown in Figure 1, the study population consisted of community dwelling patients aged 65 or older who presented to the ED after a fall or fallrelated injuries at an urban, level 1 trauma center and teaching hospital between July 2015 and April 2016. Patients were excluded if they had an altered mental status, were too unstable to consent or at baseline were unable to mobilize independently without a wheelchair. We conducted follow-up phone interviews with all patients who were referred and not referred to the outpatient fall prevention program 6 months after their fall related ED visit. This study was approved by our institutional review board and we obtained informed consent on all patients.

\section{Data collection}

The predetermined goal for this pilot study was enrollment of 100 patients. Eligible ED patients were screened and enrolled if they met the inclusion criteria and provided written informed consent. Demographics were extracted from the electronic medical record (EMR). Additionally, a semi-structured interview was conducted about their perceived health, fall risk and what the fall meant to them [10]. After 6 months, patients were contacted by telephone for an interview, which consisted of a mix of 6 closed and open-ended questions about: recurrence of falls, ED visits, current perceived health and functioning, actions undertaken to prevent falls and, if in referral group, followup with the fall prevention program (see appendix for instrument). Interviews lasted approximately 20 minutes. A maximum of 3 contact attempts were made. For the patients who could not be contacted, medical records and local obituaries were screened for recurrence of ED visits and mortality. If these measures failed, patients were deemed lost to follow-up.

\section{Measures}

Quantitative outcome measures included mortality, recurrent falls, recurrent ED visits and perceived health at 6 month follow-up. Mortality was determined if the family member reported during the follow-up call that the patient had died, if it was recorded in the EMR, or if a patient was found in a local obituary notice. Recurrent falls and ED visits were determined through follow-up calls and reviewing the EMR.

\section{Analysis}

Quantitative data were summarized using the mean and standard deviation for numerical data, categorical data were summarized using percentages. Patients' interview responses were analyzed qualitatively based on conventional content analysis methods [12]. This involved reading and re-reading the transcripts by two study investigators. Themes that emerged after the first readings were discussed and an initial coding scheme was developed. The discrepancies in the coding were then reviewed and discussed between the two study investigators to assess the multiple ways of viewing the data, illuminate blind spots and eliminate selective perceptions. Consecutively, the codes were clustered into subcategories and relationships between these were identified. The findings were separately discussed for triangulation with the principal investigator.

\section{Results}

Between July 2015 and April 2016, 100 patients were enrolled, of whom 94 patients were eligible for inclusion. Of these, 22 patients $(23 \%)$ were referred based on the zip code of their home address. As shown in the flowchart in Figure 2, for the initial ED interview 56 patients were interviewed, after which thematic saturation was reached. These

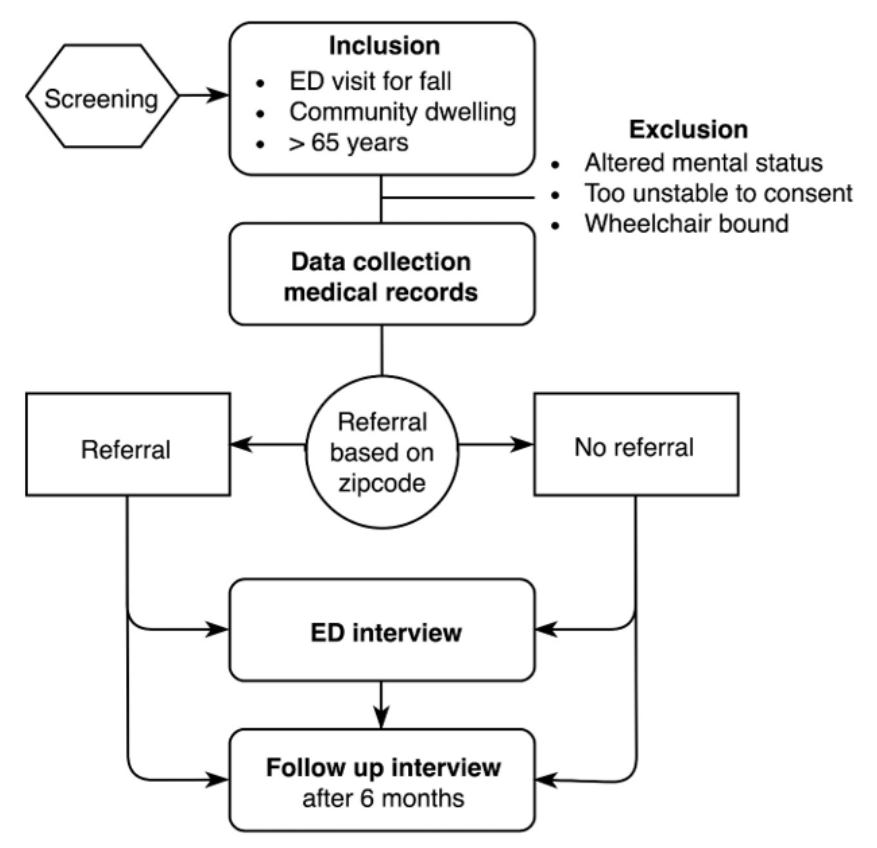

Figure 1. Study Flowchart

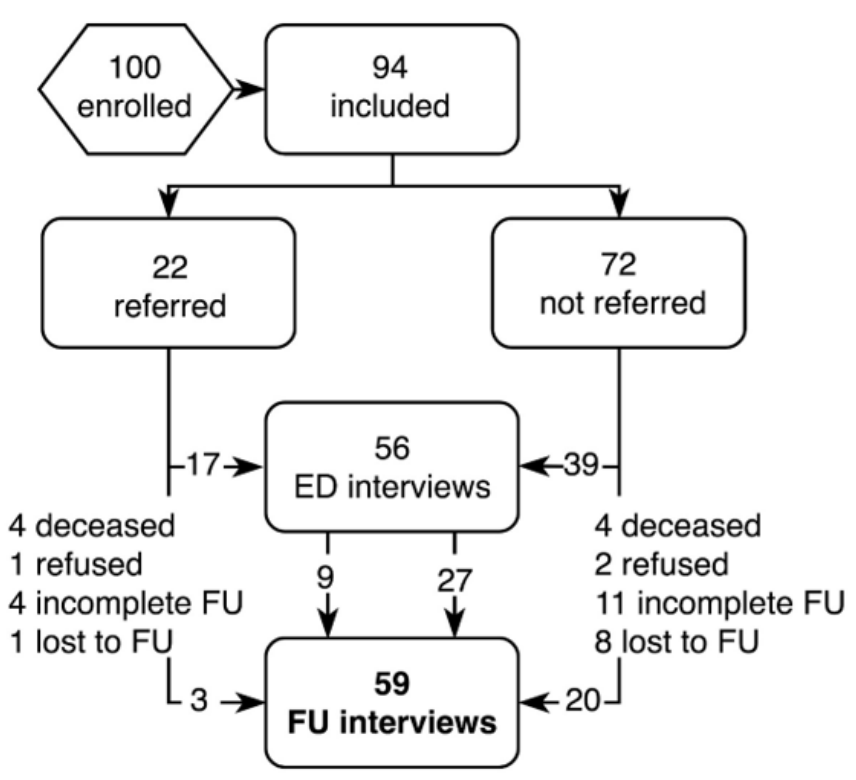

Figure 2. Inclusion Flowchart

Abbreviations: $\mathrm{ED}=$ emergency department, $\mathrm{FU}=$ follow-up 

pilot project

qualitative results assessing the attitude of patients towards their own fall risk in the ED are described in detail by Shankar, et al. [10]. At the 6 month follow-up, $59(63 \%)$ patients were interviewed and $8(8.5 \%)$ patients were deceased. The remaining 27 patients either refused $(n=3)$ or could not be contacted, of which 15 patients had notes in their EMR about subsequent hospital visits resulting in incomplete follow-up and 9 patients who were completely lost to follow up.

As shown in Table 1, there were no differences in characteristics between the referred patients in the intervention group and the control group, except from the distance of home address to the hospital (which clinic eligibility was based upon).

\section{Follow-up}

The 6-month mortality rate was $8.5 \%(n=8 / 94)$. Two $(2 / 8,25 \%)$ of these patients were re-admitted for injuries caused by a fall and died in the hospital. For 3 patients, the cause of death was unknown. Twentysix $(26 / 94,28 \%)$ patients fell again. Other outcomes 6 months after the initial fall are shown in Table 2.

None of the referred patients (0\%) participated in the falls prevention program. Two patients contacted the clinic; one declined and the other patient was referred to another program for cardiac rehabilitation. Therefore, the direct effect of the program on outcomes could not be assessed. This gave us the opportunity to explore why those at increased risk for falls do not follow up on services that could be helpful to their health and well-being.

\section{Qualitative analysis}

In the qualitative portion of the study, we explored patients' health and fall prevention behavior 6 months after their ED fall visit. Their answers reflected that there are multiple challenges to overcome in the

Table 1. Baseline characteristics

\begin{tabular}{|l|c|c|c|c|}
\hline & $\begin{array}{c}\text { Referred } \\
(\mathbf{n = 2 2})\end{array}$ & $\begin{array}{c}\text { Not referred } \\
(\mathbf{n = 7 2 )}\end{array}$ & Total (n=94) & P value \\
\hline Age, mean (SD) & $81(9.8)$ & $80(8.0)$ & $80(8.4)$ & 0.65 \\
\hline Female (\%) & $14(64 \%)$ & $57(79 \%)$ & $71(76 \%)$ & 0.14 \\
\hline $\begin{array}{l}\text { Charlson Comorbidity } \\
\text { Index, mean (SD) }\end{array}$ & $4.95(2.36)$ & $4.47(1.68)$ & $4.59(1.86)$ & 0.29 \\
\hline $\begin{array}{l}\text { Marital status married } \\
(\%)\end{array}$ & $10(46 \%)$ & $29(40 \%)$ & $39(42 \%)$ & 0.67 \\
\hline Insurance status (\%) & & & & 0.63 \\
\hline Medicare alone & $17(77 \%)$ & $60(83 \%)$ & $77(82 \%)$ & 0.83 \\
\hline Medicaid & $0(0 \%)$ & $0(0 \%)$ & $0(0 \%)$ & 0.014 \\
\hline $\begin{array}{l}\text { Education, college } \\
\text { degree or higher (\%) }\end{array}$ & $5(23 \%)$ & $18(25 \%)$ & $23(25 \%)$ & $07(311)$ \\
\hline $\begin{array}{l}\text { Distance to hospital in } \\
\text { miles, mean (SD) }\end{array}$ & $7(7.7)$ & $112(352)$ & $87)$ & \\
\hline
\end{tabular}

Table 2. Outcomes after 6 months

\begin{tabular}{|l|c|}
\hline \multicolumn{1}{|l|}{} & $\mathbf{N = 9 4}$ \\
\hline 6-month Mortality (\%) & $8(8.5 \%)$ \\
\hline Subsequent mortality for fall-related injury (\%) & $2(2.1 \%)$ \\
\hline Returned to the ED (\%) & $43(46 \%)$ \\
\hline Returned to the ED for falls (\%) & $17(18 \%)$ \\
\hline Recurrent falls (\%) & $26(28 \%)$ \\
\hline Number of falls, median (range) ${ }^{* * *}$ & $1(1-7)$ \\
\hline Reported health (\%) & \\
\hline Better & $15(25 \%)$ \\
\hline Same & $32(54 \%)$ \\
\hline Worse & $12(220 \%)$ \\
\hline
\end{tabular}

**Based on the 6-month follow-up interview, $\mathrm{N}=59$. time after the ED visit, such as 1) practical considerations, 2) competing health issues, and 3) perceptions of their fall risk.

\section{Practical considerations}

There were several practical reasons why patients did or did not manage their fall risk by going to the outpatient fall prevention program. The most frequently reported barrier by the referred patients was transportation. One of them stated: "I don't have a car and nobody is going to drive me." Another patient said, "We talked to them, but the barrier was transportation: my daughter does not drive and the program did not offer transportation." Problems with transportation were frequently mentioned in conjunction with social factors. Although per the protocol, referred patients lived in the nearby area, they often had to rely on others for transportation.

In contrast to this, a supportive partner or a social organization facilitated in modifying behavior to reduce fall risk. Two patients stated the following: "[My] wife keeps me healthy because she encourages us to do a lot of exercise. We do yoga 2 or 3 times a week. [..]" and "I'm taking Tai Chi which is excellent for balance. [...] So I started doing it. Someone came in at the senior center so I'm thrilled to have it and I believe it's helping me." A change in the social environment, such as moving to an assisted living facility, made it more difficult to adapt fall prevention behavior.

\section{Competing health priorities}

Patients reported that competing health priorities were another reason for not following up with referral or otherwise changing fall risk behavior. Several patients explained that they had to go to other therapies and treatments, such as physical therapy (PT). These treatments often had a higher priority than going to a fall prevention program. One participant stated: "I did not follow up...because I got surgery and was going through rehab. I do want to follow up with this program and I still have the brochure at home. After my shoulder surgery, I may be able to do this program." Another patient responded: "I had to go for another therapy for my vertigo."

These other types of therapies were sometimes mentioned as concomitantly reducing fall risk, although they were not specifically targeted at fall prevention. The partner of one patient summed this up: "Since the surgery he has seen a constant improvement! He has been seeing PT and OT [occupational therapist] since the surgery and they have helped him improve his balance. [...]" Other patients described doubts regarding whether it helped to prevent falls: "I thought the PT was helpful to strengthen my legs. I did it for a year and I haven't been able to get myself to do it since. I'm not sure if it helps with my balance." Another stated: "I've had home PT once a week. [...] but I still fall."

\section{Perceptions of fall risk}

Overall, there were 3 types of responses when patients were asked about fall risk: Those who did not think they were at risk, those who perceived their fall risk as a normal part of life and those who actively thought about preventive measures for their fall risk. Generally, risk denying patients in the ED described their falls as "accidents": "They were just real accidents, they just happened. So there is nothing I can do to decrease [my risk of falling]." These patients generally did not do anything to prevent falls in the following 6 months.

The second group considered themselves as at risk for falls, but only made small behavioral changes to prevent them. A view that was commonly shared amongst these patients, was that it is part of "normal" 

pilot project

life. One man who assured that he was being extra cautious and always has his cane with him since his fall, expressed his view as follows: "I'm 94 years old. I know I can't live forever. I go to the best of my ability. [...]" Another woman, who adjusted her pace to move slower to prevent falls, told she had "aches and pains" and she was "Just growing old."

The last group consisted of patients who participated in activities that are likely to reduce fall risk, although it was not initiated from a specific fall prevention program. Almost all of these patients already in the ED considered themselves to be at risk for falling and were thinking about what to do to decrease this. One woman mentioned in the followup interview that she had been taking balance classes and had put grab bars in her home, said in the ED: "[...] I should've been paying attention. Yes, and not being in such a hurry. I am aware of it when I am on stairs, particularly going down and I hold on, I mean that's the worst. For this trip to Italy I have brought balancing poles because I'm concerned if we have staircases and there is nothing to hold on to [...]."

However, the perception of fall risk seemed to evolve. Most patients at least considered being at risk for falling 6 months after the ED visit, which was not the case initially in the $\mathrm{ED}$, where the majority denied being at risk for falling.

\section{Multiple challenges to prevent falls from the ED}

The overarching theme was that there are specific challenges to effectively preventing falls in older adults by referral from the ED to a multidisciplinary prevention program. Complicating factors specific to the ED for going to a fall prevention program, are injuries from the fall that interfere with mobility and underlying conditions which contributed to the fall. These all require attention from the patient and their family. Coping with a fall physically and psychosocially adds to the barriers for older adults to go to a fall prevention program.

\section{Discussion and conclusion}

This pilot study demonstrated a zero follow-up rate after an ED based referral to a multidisciplinary fall prevention program, with only two patients contacting the clinic and no patients participating in the program. Nevertheless, qualitative analyses of follow-up interviews revealed unexpected challenges which could aid the implementation future ED based referral to preventive interventions. The time after the ED visit for fall related injuries was characterized by practical challenges, competing health priorities and changes in perception of their own fall risk.

Although it can be argued that established and widely accepted treatments such as PT often simultaneously address issues that are also part of a fall prevention program, the preventive effect on falls appears variable and unreliable.

During the follow-up interviews 6 months after the fall, many patients expressed reflections on their own fall risk, which was different from their ED interviews. This could mean that the ED provides the opportunity to identify patients who might benefit from referral to a fall prevention program, even if the patient initially denies being at risk for falls. Unfortunately, in the fast-paced ED environment, referral alone did not result in follow up with the fall prevention program. A similar study where patients received a flyer in the ED for a comparable fall prevention program, also reported zero follow up in clinic 60 days post ED visit [11].

Given our patients had a significant mortality rate $(8.5 \%)$ and falls recurrence rate $(28 \%)$ overcoming the challenges to preventing falls is important. While these numbers are similar to those previously reported [3], our figures are still likely to be an underestimate, as the patients who could not be contacted may have fallen again without presenting to our hospital's ED.

Given the substantial risk of recurrence of falls in geriatric patients, an extra effort must be made for effective referral to for fall prevention programs. This effort could be aimed at overcoming practical barriers in terms of transportation, helping to make appointments that do not conflict with other competing health priorities, and integration with other already established therapies. Also. appropriate education about fall risk and prevention could help [13]. Since visiting the ED after a fall is likely a stressful event itself, the timing and the ED-setting could possibly be suboptimal for referral. A study from England with similar inclusion criteria, contacted patients by mail directly and by telephone 2-3 days after an ED visit for a fall. The participation in the intervention group was much higher with $77 \%$, which resulted $50 \%$ fracture reduction caused by recurrence of falls [14]. A study about follow-up after a fragility fracture with an osteoporosis intervention, found a trend towards better follow-up with a more personalized approach with a phone call in addition to an invitation letter [15]. Therefore, contacting patients a few days after the ED visit, might result in higher follow-up rates for fall prevention interventions and thereby less morbidity and mortality caused by falls.

There were several limitations to this study. The largest limitation of this study is that there are patients who did not complete the follow-up interview at 6 months. There were also patients who were completely lost to follow-up, without the possibility to retrieve additional information about vital status or recurrence of falls. This could have introduced bias in the quantitative results although we minimized this by following up through the EMR and obituaries. Furthermore, there is always the risk of patients' answers being affected by social desirability bias. However, in this case, many answers did not seem to support extensive changes in behavior.

\section{Conclusion}

In conclusion, this pilot study revealed unexpected challenges for ED based referral to a fall prevention program for older adults. Unfortunately, referral alone is not enough to effectively prevent falls for older adults presenting in the ED after a fall. Actively addressing barriers to fall clinic follow-up is necessary to improve outpatient fall clinic show rates.

\section{Funding}

This research received no specific grant from any funding agency in the public, commercial, or not-for-profit sectors.

\section{Conflicting Interests}

The authors declare that there is no conflict of interest.

\section{Supplementary materials}

A detailed description about the fall prevention program is available online at http://spauldingrehab.org/conditions-and-treatments/livelong-walk-strong The interview instruments and responses are available upon request with the corresponding author.

\section{Authorship}

All authors made substantial contributions. TR and SL conceived of the study, NT and CR collected the data. SV and NT analyzed the data. 
van Wijck SFM (2019) Unexpected challenges to preventing falls in older adults: a mixed methods study of an emergency department-based falls prevention referral pilot project

SV wrote the original draft. SL, TR, NT and CR edited the manuscript. SL supervised the entire study.

\section{References}

1. Ten leading causes of death and injury (2015) Centers for Disease Control and Prevention.

2. Burns ER, Stevens JA, Lee R (2016) The direct costs of fatal and non-fatal falls among older adults- United States. J Safety Res 58: 99-103.

3. Liu SW, Obermeyer Z, Chang Y, Shankar KN (2015) Frequency of ED revisits and death among older adults after a fall. Am J Emerg Med 33: 1012-1018. [Crossref]

4. Panel on Prevention of Falls in Older Persons AGS, British Geriatrics S (2011) Summary of the Updated American Geriatrics Society/British Geriatrics Society clinical practice guideline for prevention of falls in older persons. $J$ Am Geriatr Soc 59: 148-157. [Crossref]

5. Tricco AC, Thomas SM, Veroniki AA, Haid JS, Cogo E, et al. (2017) Comparisons of Interventions for Preventing Falls in Older Adults: A Systematic Review and Metaanalysis. J Amer Med Asso 318: 1687-1699.

6. Gillespie L, Handoll H (2009) Prevention of falls and fall-related injuries in older people. Inj Prev 15: 354-355.

7. Child S, Goodwin V, Garside R, Jones-Hughes T, Boddy K, et al. (2012) Factors influencing the implementation of fall-prevention programmes: a systematic review and synthesis of qualitative studies. Implement Sci 7: 91. [Crossref]
8. Schoon Y, Hoogsteen-Ossewaarde ME, Scheffer AC, Van Rooij FJ, Rikkert MG, et al. (2011) Comparison of different strategies of referral to a fall clinic: how to achieve an optimal casemix? J Nutr Health Aging 15: 140-145. [Crossref]

9. Sanders KM, Lim K, Stuart AL, Macleod A, Scott D, et al. (2017) Diversity in fall characteristics hampers effective prevention: the precipitants, the environment, the fall and the injury. Osteoporos Int 28: 3005-3015.

10. Shankar KN, Taylor D, Rizzo CT, Liu SW (2017) Exploring Older Adult ED Fall Patients' Understanding of Their Fall: A Qualitative Study. Geriatr Orthop Surg Rehabil 8: 231-237.

11. Shankar KN, Treadway NJ, Taylor AA, Breaud AH, Peterson EW, et al. (2017) Older adult falls prevention behaviors 60 days post-discharge from an urban emergency department after treatment for a fall. Inj Epidemiol 4: 18.

12. Hsieh HF, Shannon SE (2005) Three approaches to qualitative content analysis. Qual Health Res 15: 1277-1288. [Crossref]

13. Yardley L, Beyer N, Hauer K, McKee K, Ballinger C, et al. (2007) Recommendations for promoting the engagement of older people in activities to prevent falls. Qual Saf Health Care. 16: 230-234. [Crossref]

14. Close J, Ellis M, Hooper R, Glucksman E, Jackson S, et al. (1999) Prevention of falls in the elderly trial (PROFET): a randomised controlled trial. Lancet 353: 93-97. [Crossref]

15. O'Brien LK, Armstrong AD, Hassenbein SE, Fox EJ (2015) Evaluation of Patients' Response Toward Osteoporosis Letter Intervention Versus Phone Call Plus Letter Intervention. Geriatr Orthop Surg Rehabil 6: 246-250.

Copyright: $(2019$ van Wijck SFM. This is an open-access article distributed under the terms of the Creative Commons Attribution License, which permits unrestricted use, distribution, and reproduction in any medium, provided the original author and source are credited. 